\title{
A Real-Time Pothole Detection Approach for Intelligent Transportation System
}

\author{
Hsiu-Wen Wang, ${ }^{1}$ Chi-Hua Chen, ${ }^{2}$ Ding-Yuan Cheng, ${ }^{3}$ Chun-Hao Lin, ${ }^{2}$ and Chi-Chun Lo ${ }^{1}$ \\ ${ }^{1}$ Institute of Information Management, National Chiao Tung University, Hsinchu 300, Taiwan \\ ${ }^{2}$ Telecommunication Laboratories, Chunghwa Telecom Co., Ltd., Taoyuan 326, Taiwan \\ ${ }^{3}$ Department of Information Management, Hwa Hsia University of Technology, New Taipei 235, Taiwan
}

Correspondence should be addressed to Chi-Hua Chen; chihua0826@gmail.com

Received 14 August 2014; Revised 4 December 2014; Accepted 9 December 2014

Academic Editor: Jung-Fa Tsai

Copyright (C) 2015 Hsiu-Wen Wang et al. This is an open access article distributed under the Creative Commons Attribution License, which permits unrestricted use, distribution, and reproduction in any medium, provided the original work is properly cited.

\begin{abstract}
In recent years, fast economic growth and rapid technology advance have led to significant impact on the quality of traditional transport system. Intelligent transportation system (ITS), which aims to improve the transport system, has become more and more popular. Furthermore, improving the safety of traffic is an important issue of ITS, and the pothole on the road causes serious harm to drivers' safety. Therefore, drivers' safety may be improved with the establishment of real-time pothole detection system for sharing the pothole information. Moreover, using the mobile device to detect potholes has been more popular in recent years. This approach can detect potholes with lower cost in a comprehensive environment. This study proposes a pothole detection method based on the mobile sensing. The accelerometer data is normalized by Euler angle computation and is adopted in the pothole detection algorithm to obtain the pothole information. Moreover, the spatial interpolation method is used to reduce the location errors from global positioning system (GPS) data. In experiments, the results show that the proposed approach can precisely detect potholes without false-positives, and the higher accuracy is performed by the proposed approach. Therefore, the proposed real-time pothole detection approach can be used to improve the safety of traffic for ITS.
\end{abstract}

\section{Introduction}

In recent years, fast economic growth and rapid technology advance have led to significant impact on the quality of traditional transport system. Intelligent transportation system (ITS), which aims to improve the transport system, has become more and more popular. For the safety of traffic, road users often feel uncomfortable when they drive on the rough roads, especially the potholes on the road. In accordance with the statistics from the Ministry of Justice in Taiwan, the national compensation money is about 240 million dollars from 2008 to 2011 [1]. The pothole on the road causes serious harm to drivers' safety. Therefore, drivers' safety may be improved with the establishment of real-time pothole detection system for sharing the pothole information.

Moreover, more and more sensors which include $G$ sensors, electronic compass, gyroscope, global positioning system (GPS), microphone, and cameras are equipped in mobile device (e.g., smartphone and iPad). Several applications use these sensors in mobile devices and combine mobile sensing techniques to solve problems such as social network [2], healthcare [3], environment monitoring [4], and traffic information [5]. Therefore, using the mobile device based on mobile sensing techniques to detect potholes is suitable and convenient.

This study proposes a pothole detection method based on the mobile sensing and shares the pothole information with road users and government. For this purpose, the mobile device should be equipped with $G$-sensors and GPS to collect accelerometer data and location information. The accelerometer data is normalized by Euler angle computation and is adopted in the pothole detection algorithm to obtain the pothole information. Moreover, the spatial interpolation method is used to reduce the location errors from GPS data. Then the pothole information is made public to improve the safety of traffic. 
The remainder of the paper is organized as follows. Section 2 presents and discusses the various techniques of image recognition method and mobile sensing method for pothole detection. In Section 3, this study proposes a realtime pothole detection method based on mobile sensing. This study also illustrates the experiment results and analyses in Section 4. Finally, this study concludes the paper in Section 5.

\section{Related Work}

Some pothole detection methods have been proposed and can be classified into two groups: image recognition method and mobile sensing method. The advantages and shortcomings of these methods are presented in the following subsections.

2.1. Image Recognition Method. Yu and Salari proposed a pothole detection approach based on laser imaging techniques to collect road information. Then the artificial neural network algorithm (ANN) was used to analyze the road information and detect potholes [6]. However, this approach which requires a big computation power to recognize the laser images is unsuitable for mobile devices.

Lin and Liu used the support vector machine algorithm (SVM) to analyze images about road information for pothole detection [7]. Although this approach can provide high accuracy, a big computation power is required for image recognition. Therefore, this approach is also unsuitable for mobile devices.

2.2. Mobile Sensing Method. For BusNet project, the Gsensor and GPS are equipped in the on-board unit (OBU) in bus to collect accelerometer data and location information. These data can be sent to data processing center via wireless networks, and data processing center can analyze these data to check whether the vectors of accelerometer data exceed the thresholds for pothole detection [8]. However, this approach requires that the batch accelerometer data is sent when bus enters the bus station. Therefore, this approach cannot provide real-time pothole detection information.

The pothole patrol system which was proposed by a project team from Massachusetts Institute of Technology combined G-sensor and GPS. This system analyzed the $x$ axis accelerometer data and $z$-axis accelerometer data and equipped five data filters which include (1) speed, (2) highpass, (3) $z$-peak, (4) $x z$-ratio, and (5) speed versus $z$ ratio [9]. Although these data filters can detect potholes, only $z$ peak of data filter can obtain the precise pothole information. However, high misjudgment of $z$-peak of data filters with the surge of road.

Nericell project used a smartphone based on Windows Mobile operation system which is equipped with $G$-sensor and GPS to collect and analyze accelerometer data for pothole detection [10]. However, the smartphone in this project should be equipped with the specific angle. Furthermore, this project only considered analyzing $z$-axis accelerometer data with high misjudgment.
Mednis et al. proposed four pothole detection approaches which include (1) Z-THRESH approach, (2) Z-DIFF approach, (3) STDEV-Z approach, and (4) G-ZERO approach to analyze the accelerometer data [11]. The accelerometer data in this study is obtained from Tmote sensors, Texas Instruments controllers, and Analog Devices G-sensors [12]. However, the results of Z-THRESH approach and G-ZERO approach would be influenced by peak value to generate more false-positives. The results of $Z$-DIFF approach and STDEV- $Z$ approach are dependent on frequency and timing. Therefore, the design and comparisons of these approaches for mobile device require to be investigated.

2.3. Summary. In summary, due to the requirement of big computation power for image recognition method, too many resources are allocated for this method to be an efficiency mobile device. Therefore, mobile sensing method is more suitable to detect potholes for mobile device. However, previous pothole detection approaches based on mobile sensing only considered one threshold to detect pothole, and high false-positives are obtained from these approaches. Consequently, this study considers Mednis's approaches [11] and proposes a real-time pothole detection method to improve the accuracy of pothole detection method.

\section{Real-Time Pothole Detection Method}

The proposed real-time pothole detection method based on mobile sensing includes three steps: (1) accelerometer data normalization, (2) pothole detection approaches, and (3) pothole location determination.

3.1. Problem Definition. Some shortcomings are in previous pothole detection methods as follows: (1) mobile device should be equipped with the specific angle; (2) high falsepositives may be generated with considering only one threshold for pothole detection; (3) the precise pothole location has not been investigated.

Therefore, this study proposes a real-time pothole detection method based on mobile sensing to collect and normalize the accelerometer data from mobile device for free angle establishment. Furthermore, a pothole detection algorithm is proposed to consider several thresholds and combine several pothole detection approaches for pothole detection accuracy improvement. Finally, the space interpolation method is adopted to determine pothole location for leaving shortcomings.

3.2. Accelerometer Data Normalization. For solving the limitation of the specific angle in previous pothole detection approaches, this study uses Euler angle formulas to normalize the three-axis accelerometer data. The Euler angles describe the vector set in 3-dimensional Euclidean space three parameters and represent a sequence of three elemental rotations. For example, Figure 1 shows that the vector set of accelerometer data is defined as $\left\{x^{\prime}, y^{\prime}, z^{\prime}\right\}$. The $x^{\prime} y^{\prime} z^{\prime}$ system rotates about the $x^{\prime}$-axis by angle $\alpha$. The $y^{\prime}$-axis is now at angle $\alpha$ with respect to the $y$-axis, and the $z^{\prime}$-axis is now at angle $\alpha$ with respect to the $z$-axis. In accordance with 


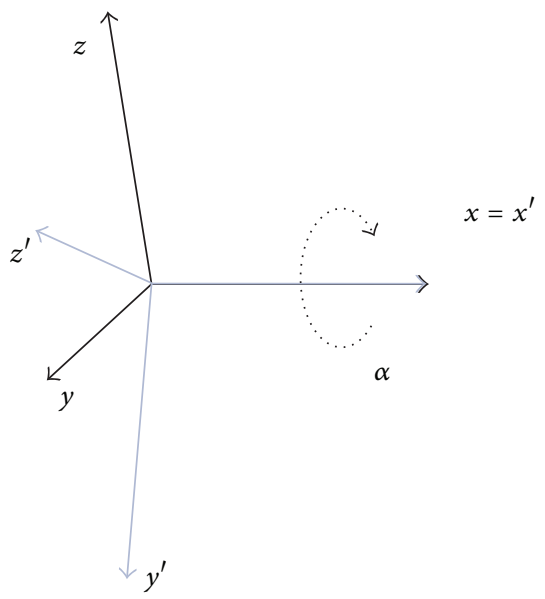

Figure 1: A case study of Euler angles (the $x^{\prime} y^{\prime} z^{\prime}$ system rotates about the $x^{\prime}$-axis by angle $\alpha$ ).

Euler angle formulas, the vector set $\{x, y, z\}$ can be calculated by adopting the values of vector set $\left\{x^{\prime}, y^{\prime}, z^{\prime}\right\}$ and angle $\alpha$ (shown in formulas (1)). Furthermore, the vector of each axis can be calculated by using Euler angle formulas when the system rotates about the $y^{\prime}$-axis by angle $\beta$ and $z^{\prime}$-axis by angle $\gamma$ (shown in formulas (2)). Therefore, the vector of each axis with 0 degree angle is referred to as baseline in this study. In runtime stage, the vector set $\{x, y, z\}$ can be calculated by adopting the vector set of baseline and rotation angle for accelerometer data normalization:

$$
\begin{aligned}
& {\left[\begin{array}{lll}
x & y & z
\end{array}\right]=\left[\begin{array}{lll}
x^{\prime} & y^{\prime} & z^{\prime}
\end{array}\right]\left[\begin{array}{ccc}
1 & 0 & 0 \\
0 & \cos \alpha & -\sin \alpha \\
0 & \sin \alpha & \cos \alpha
\end{array}\right]} \\
& x=x^{\prime}, \\
& y=y^{\prime} \times(\cos \alpha)+z^{\prime} \times(\sin \alpha), \\
& z=y^{\prime} \times(-\sin \alpha)+z^{\prime} \times(\cos \alpha), \\
& {\left[\begin{array}{lll}
x & y & z
\end{array}\right]=\left[\begin{array}{lll}
x^{\prime} & y^{\prime} & z^{\prime}
\end{array}\right]\left[\begin{array}{ccc}
\cos \beta & 0 & \sin \beta \\
0 & 1 & 0 \\
-\sin \beta & 0 & \cos \beta
\end{array}\right] \text {, }} \\
& x=x^{\prime} \times(\cos \beta)+z^{\prime} \times(-\sin \beta) \text {, } \\
& y=y^{\prime}, \\
& z=x^{\prime} \times(\sin \beta)+z^{\prime} \times(\cos \beta), \\
& {\left[\begin{array}{lll}
x & y & z
\end{array}\right]=\left[\begin{array}{lll}
x^{\prime} & y^{\prime} & z^{\prime}
\end{array}\right]\left[\begin{array}{ccc}
\cos \gamma & -\sin \gamma & 0 \\
\sin \gamma & \cos \gamma & 0 \\
0 & 0 & 1
\end{array}\right]} \\
& x=x^{\prime} \times(\cos \gamma)+y^{\prime} \times(\sin \gamma), \\
& y=x^{\prime} \times(-\sin \gamma)+y^{\prime} \times(\cos \gamma), \\
& z=z^{\prime} \text {. }
\end{aligned}
$$

(1)

3.3.2. The Second Pothole Detection Approach: Z-DIFF. The $Z$-DIFF approach considers the maximum difference of two consecutive $z$-axis accelerometer records as the threshold to detect pothole. Due to much decreasing and increasing of $z$-axis accelerometer data through a pothole, the velocity of variation of $z$-axis accelerometer data between time $t_{i, j-1}$ and time $t_{i, j}$ is calculated and used to detect pothole. Therefore, this study retrieves the largest value of velocity of variation of $z$-axis accelerometer data through a pothole in experimental runs. Furthermore, the minimum value of the maximum value of variation velocity in each run is selected as the threshold $\theta_{2}$ which is suitable to detect pothole for each experimental run (shown in formula (5)). In runtime stage, the value of $f_{2}\left(g_{a, i, j}\right)$ is 1 when the value of $\mid g_{a, i, j}-$ $g_{a, i, j-1} \mid /\left(t_{i, j}-t_{i, j-1}\right)$ is larger than $\theta_{2}$ for pothole detection (shown in formula (6)). However, the limitation of this approach is difficult to determine the time difference between $t_{i, j-1}$ and $t_{i, j}$, and the accuracy of this approach is influenced by this time difference.

The value of threshold is

$$
\theta_{2}=\min _{a=1,1 \leq i \leq n, i \in N} \max _{e_{i} \leq j \leq l_{i}, j \in N} \frac{\left|g_{a, i, j}-g_{a, i, j-1}\right|}{t_{i, j}-t_{i, j-1}} .
$$


Detection function is

$$
f_{2}\left(g_{a, i, j}\right)= \begin{cases}1, & \text { if } \frac{\left|g_{a, i, j}-g_{a, i, j-1}\right|}{t_{i, j}-t_{i, j-1}} \geq \theta_{2} \\ 0, & \text { others, }\end{cases}
$$

where $a=1, \quad 1 \leq i \leq n, \quad i \in N, \quad j \geq 1, \quad j \in N$.

3.3.3. The Third Pothole Detection Approach: STDEV(Z). The $\operatorname{STDEV}(Z)$ approach considers the maximum standard deviation of $z$-axis accelerometer data as the threshold to detect pothole. Due to the perturbation motion of $z$-axis accelerometer data through a pothole, the standard deviation of $z$-axis accelerometer data during $K$ records is calculated and used to detect pothole. Therefore, this study gets the maximum value of standard deviation of $z$-axis accelerometer data through a pothole in experimental runs. Furthermore, the minimum value of the maximum value of standard deviation in each run is selected as the threshold $\theta_{3}$ which is suitable to detect pothole for each experimental run (shown in formula (7)). In runtime stage, the value of $f_{3}\left(g_{a, i, j}\right)$ is 1 when the value of $\sqrt{\sum_{k=j-K+1}^{j}\left(g_{a, i, k}-\mu_{i, j}\right)^{2} / K}$ is larger than $\theta_{3}$ for pothole detection (shown in formula (8)). However, the limitation of this approach is difficult to determine the value of $K$ which means time period, and the accuracy of this approach is influenced by this time period.

The value of threshold is

$$
\begin{array}{r}
\theta_{3}=\min _{a=1,1 \leq i \leq n, i \in N} \max _{e_{i} \leq j \leq l_{i}, j \in N} \sqrt{\frac{\sum_{k=j-K+1}^{j}\left(g_{a, i, k}-\mu_{i, j}\right)^{2}}{K}}, \\
\text { where } \mu_{i, j}=\frac{\sum_{k=j-K+1}^{j} g_{a, i, k}}{K} .
\end{array}
$$

Detection function is

$$
f_{3}\left(g_{a, i, j}\right)= \begin{cases}1, & \text { if } \sqrt{\frac{\sum_{k=j-K+1}^{j}\left(g_{a, i, k}-\mu_{i, j}\right)^{2}}{K}} \geq \theta_{3} \\ 0, & \text { others, }\end{cases}
$$

where $a=1, \quad 1 \leq i \leq n, \quad i \in N, \quad j \geq K$,

$$
j \in N, \quad \mu_{i, j}=\frac{\sum_{k=j-K+1}^{j} g_{a, i, k}}{K} .
$$

3.3.4. The Fourth Pothole Detection Approach: G-ZERO. The $G$-ZERO approach considers all three-axis accelerometer data and selects a lower bound and upper bound to detect pothole for accuracy improvement. When a car passes through a pothole, all values of three-axis accelerometer data are near to zero. Therefore, this study considers the largest value of three-axis accelerometer data through a pothole as a candidate of lower bound and the lowest value of threeaxis accelerometer data through a pothole as a candidate of upper bound. Then the minimum value of the maximum value of three-axis accelerometer data in each run is selected as the lower bound $\theta_{4,1}$, and the maximum value of the minimum value of three-axis accelerometer data in each run is selected as the upper bound $\theta_{4,2}$ (shown in formulas (9) and (10)). In runtime stage, the value of $f_{4}\left(g_{a, i, j}\right)$ is 1 when the value of $g_{a, i, j}$ is larger than $\theta_{4,1}$ and is lower than $\theta_{4,2}$ for pothole detection (shown in formula (11)).

The value of lower bound is

$$
\theta_{4,1}=\min _{a \in\{1,2,3\}, 1 \leq i \leq n, i \in N} \max _{e_{i} \leq j \leq l_{i}, j \in N} g_{a, i, j} .
$$

The value of upper bound is

$$
\theta_{4,2}=\max _{a \in\{1,2,3\}, 1 \leq i \leq n, i \in N} \min _{e_{i} \leq j \leq l_{i}, j \in N} g_{a, i, j} .
$$

Detection function is

$$
f_{4}\left(g_{a, i, j}\right)= \begin{cases}1, & \text { if } \theta_{4,1} \leq g_{a, i, j} \leq \theta_{4,2} \\ 0, & \text { others }\end{cases}
$$

where $a=\{1,2,3\}, \quad 1 \leq i \leq n, \quad i \in N, \quad j \in N$.

3.3.5. The Fifth Pothole Detection Approach: Proposed Approach. This subsection proposes a pothole detection approach which combines and improves the Z-THRESH and G-ZERO approaches to detect pothole. Furthermore, the $Z$-DIFF and $\operatorname{STDEV}(Z)$ approaches are limited in accordance with time differences and time periods, so these two approaches are not adopted. The pseudocode of the proposed pothole detection approach is presented in Algorithm 1. The input parameters of this proposed approach are three-axis accelerometer data, and the value of output is 1 when the proposed pothole detection approach supposes the car passed through a pothole. In the proposed approach, the parameter check_method is used to record whether the value of $f_{1}\left(g_{a, i, j}\right)$ or $f_{4}\left(g_{a, i, j}\right)$ is 1 . When one of Z-THRESH and $G$-ZERO approaches supposes that the car passed through a pothole, the timestamp $t_{i, j}$ is recorded and compared with the parameter check_time. The value of output is 1 if $t_{i, j}$-check_time is smaller than $\varepsilon$ seconds, which means a pothole is detected. Furthermore, the parameter check_time can be trained and learned by historical data from each practical run.

3.4. Pothole Location Determination. For pothole location determination, this study uses the space interpolation method to obtain precise pothole location. Figure 2 shows that two locations (i.e., $L_{1}$ and $L_{2}$ ) and timestamps (i.e., $t_{1}$ and $t_{2}$ ) obtained from GPS module are adopted in the space interpolation method to determine the pothole location. The function $d\left(L_{1}, L_{2}\right)$ is defined as the distance between location $L_{1}$ and location $L_{2}$. Therefore, the pothole location $L_{p}$ can be determined by using

$$
\begin{aligned}
& d\left(L_{1}, L_{p}\right)=\frac{d\left(L_{1}, L_{2}\right) \times\left(t_{2}-t_{1}\right)}{\left(t_{3}-t_{1}\right)}, \\
& d\left(L_{2}, L_{p}\right)=\frac{d\left(L_{1}, L_{2}\right) \times\left(t_{3}-t_{2}\right)}{\left(t_{3}-t_{1}\right)} .
\end{aligned}
$$




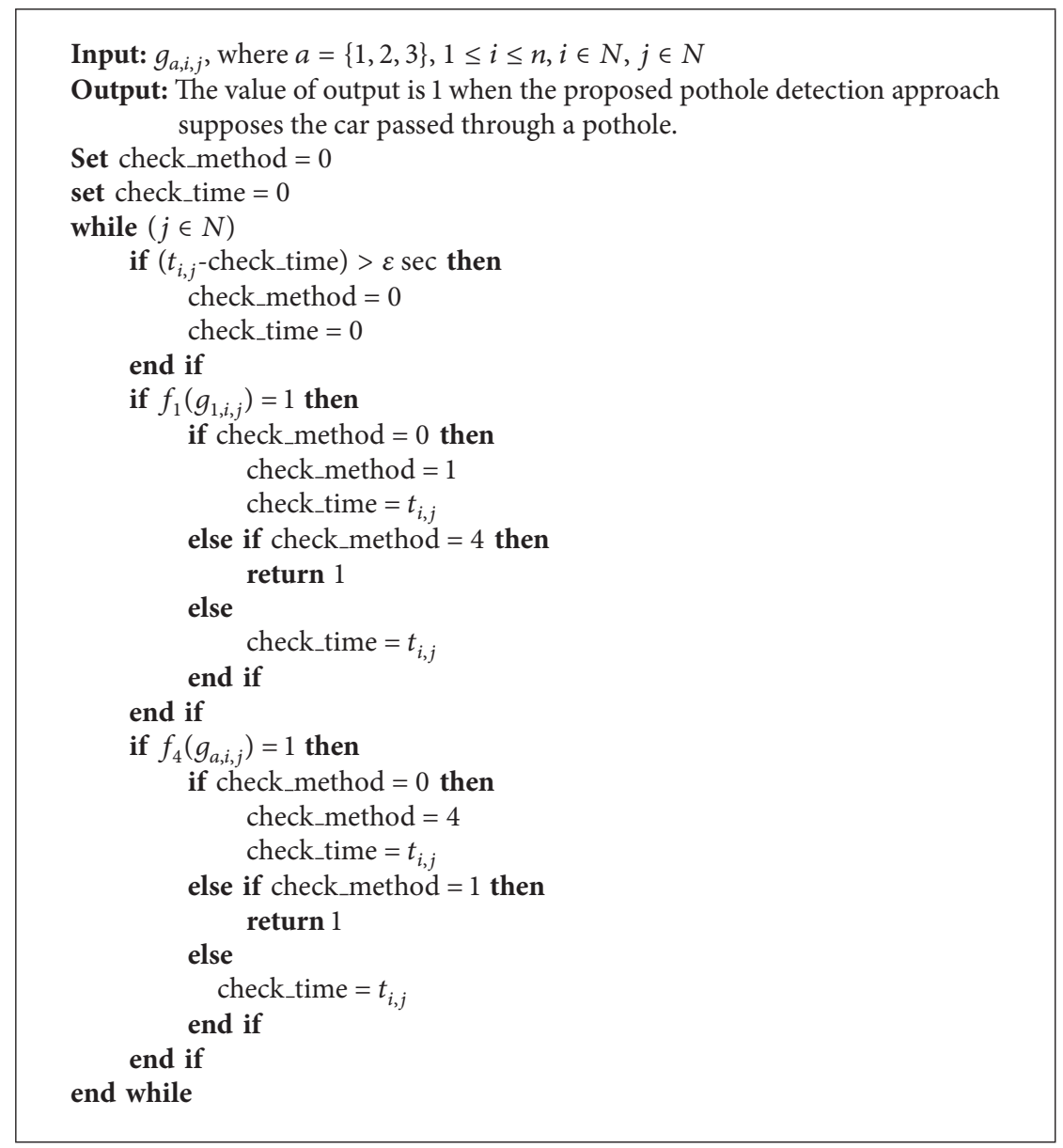

Algorithm 1: The pseudocode of the proposed pothole detection approach.

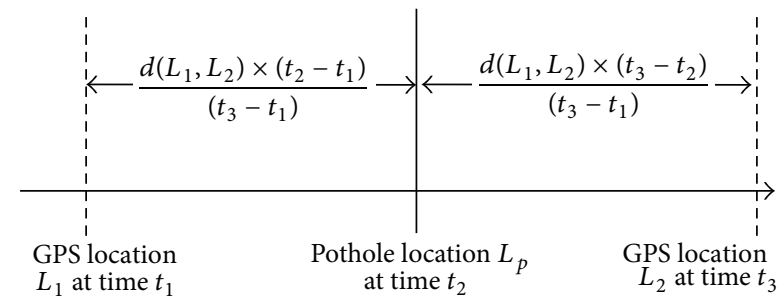

FIgURE 2: The space interpolation method for pothole location determination.

\section{Experimental Results}

This section discusses the analyses of experimental results for accelerometer data normalization, pothole detection approaches, and pothole location determination.

4.1. The Analyses of Accelerometer Data Normalization. For the analyses of accelerometer data normalization, this study gives two case studies which include (1) the mobile device with 0 degree angle as baseline (shown in Figure 3) and (2) the mobile device with -15 degree angle (i.e., the value of $\alpha$ is -15 in Figure 1) (shown in Figure 4). Then the $t$-test and $F$-test are

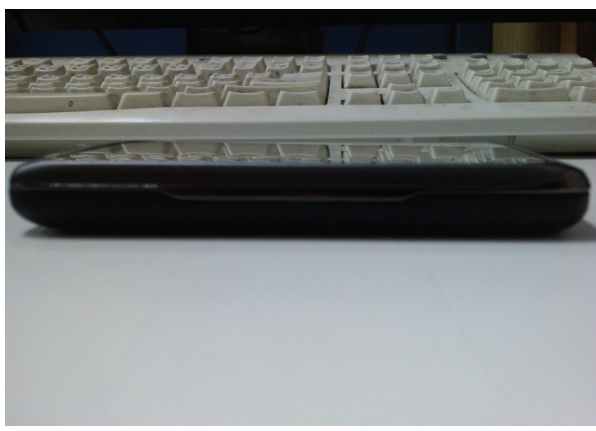

FIGURE 3: A case study of the mobile device with 0 degree angle as baseline.

used to verify the difference between the $z$-axis accelerometer data of baseline in Case 1 and the $z$-axis accelerometer data after accelerometer data normalization in Case 2.

This study uses two-tailed $t$-test to determine significance of the difference between the mean of $z$-axis accelerometer data of baseline in Case $1\left(\mu_{1}=-9.8489\right)$ and the mean of $z$ axis accelerometer data after accelerometer data normalization in Case $2\left(\mu_{2}=-9.8476\right)$. The sample sizes of Case 1 and Case 2 are 60. Furthermore, this study also uses $F$-test to 


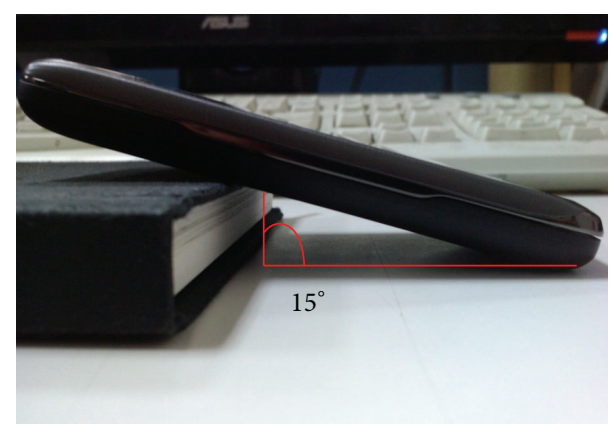

FIgURE 4: A case study of the mobile device with -15 degree angle.

determine significance of the difference between the variance of $z$-axis accelerometer data of baseline in Case $1\left(\sigma_{1}{ }^{2}=\right.$ $0.000077)$ and the variance of $z$-axis accelerometer data after accelerometer data normalization in Case $2\left(\sigma_{2}^{2}=0.000085\right)$. Table 1 shows the $t$-test and $F$-test for the population means and variances of samples in Case 1 and Case 2. Experimental results indicate that the null hypothesis $\left(\mathrm{H} 0: \mu_{1}=\mu_{2}\right)$ in $t$ test is accepted, and another null hypothesis $\left(\mathrm{H} 0: \sigma_{1}=\sigma_{2}\right)$ in $F$-test is also accepted. Therefore, using Euler angle formulas to normalize the accelerometer data from mobile device is suitable for free angle establishment.

4.2. The Accuracy of Pothole Detection Approach. For the analyses of accuracy of pothole detection approach, this study selects a pothole (length: $58 \mathrm{~cm}$; weight: $51 \mathrm{~cm}$; and depth: $6 \mathrm{~cm}$ ) as a case study (shown in Figure 5) and 10 runs in experiment environment. The mean frequency of accelerometer data detection in $G$-sensor in mobile device is 124 counts/second. The $k$-fold cross-validation [13] is used to verify the accuracy of pothole detection approach. In experiments, training and testing are performed 10 times (i.e., $k=$ 10). In iteration $i$, the accelerometer data in $i$ th run therapy is selected as the test corpus, and the accelerometer data in other runs is collectively used to train the thresholds for each approach. Table 2 shows the comparisons of $Z$-THRESH approach, $Z$-DIFF approach, STDEV $(Z)$ approach, G-ZERO approach, and the proposed approach. The results show that the proposed approach can precisely detect potholes without false-positives and the accuracy of the proposed approach is $100 \%$. Furthermore, this study also implemented and compared common machine learning methods which include ANN, SVM, and decision tree (DT) (shown in Table 3). Although these machine learning methods can detect potholes, several false-positives are generated by them.

4.3. The Error of Pothole Location Determination. For the analyses of error of pothole location determination, this study uses the accelerometer data and location information from 10 runs in Section 4.2 to verify the space interpolation method. The results show that the error of pothole location determination is reduced from 17.47 meters to 11.74 meters after using the space interpolation method. Therefore, the space interpolation method is suitable to determinate the precise pothole location.

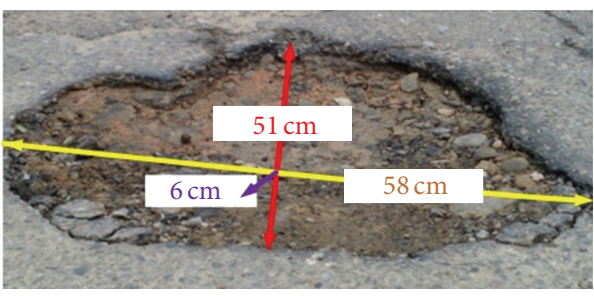

FIgURE 5: A case study of a pothole in University Road, Hsinchu, Taiwan.

TABLE 1: The test results for accelerometer data with normalization.

\begin{tabular}{lc}
\hline & $\begin{array}{c}\text { The value of } z \text {-axis accelerometer data } \\
\text { mean (standard deviation) }\end{array}$ \\
\hline $\begin{array}{l}\text { The } 0 \text { degree angle } \\
\text { (baseline) }\end{array}$ & $-9.8489(0.000077)$ \\
$\begin{array}{l}\text { The }-15 \text { degree angle after } \\
\text { normalization }\end{array}$ & $-9.8476(0.000085)$ \\
\hline
\end{tabular}

TABLE 2: The false positive of each pothole detection approach.

\begin{tabular}{lccccc}
\hline $\begin{array}{l}\text { Detection } \\
\text { method }\end{array}$ & $Z$-THRESH & $Z$-DIFF & STDEV $(Z)$ & $G$-ZERO & $\begin{array}{c}\text { Proposed } \\
\text { method }\end{array}$ \\
\hline $\begin{array}{l}\text { False- } \\
\text { positive }\end{array}$ & 49 & 40 & 274 & 8 & 0 \\
\hline
\end{tabular}

TABLE 3: The comparisons of different machine learning methods.

\begin{tabular}{lccc}
\hline Detection method & ANN & SVM & DT \\
\hline False-positive & 1626 & 255 & 282 \\
\hline
\end{tabular}

\section{Conclusions and Future Work}

This study proposes a real-time pothole detection method based on the mobile sensing techniques. This method uses Euler angle computation to normalize the accelerometer data obtained from mobile device with free angle establishment. Moreover, a pothole detection approach is proposed to be combined with Z-THRESH and G-ZERO approaches for reducing the false-positives of pothole detection. Furthermore, the spatial interpolation method is adopted to obtain precisely the location of pothole. In experiments, the results show that the proposed approach can precisely detect potholes without false-positives and the accuracy of the proposed approach is $100 \%$. Therefore, the proposed realtime pothole detection approach can be used to improve the safety of traffic for ITS.

However, the limitation of this study is sample size. In the future, more practical results will be retrieved and analyzed to deploy the proposed method everywhere. Furthermore, due to the limited battery capacity of mobile device, the issue about saving of computation power can be investigated. A green pothole detection approach is needed to reduce the frequency of accelerometer data detection with high accuracy of pothole detection. 


\section{Notations}

$g_{a, i, j}:$ The acceleration of the $a$ th axle of the $j$ th record in the $i$ th run

$t_{i, j}$ : The timestamp of the $j$ th record in the $i$ th run

$n: \quad$ The number of runs

$e_{i}$ : The $e_{i}$ th entering pothole record in the $i$ th run

$l_{i}$ : The $l_{i}$ th leaving pothole record in the $i$ th run

a: The coordinate axis of $G$-sensor in mobile device (e.g., the value of $a$ is 1 which means $Z$-axis)

$f_{m}(\cdot)$ : The output of the $m$ th pothole detection approach (e.g., the value of $f_{1}(\cdot)$ is 1 when the first pothole detection approach supposes that the car passed through a pothole)

K: $\quad$ The third pothole detection approach requires $K$ records to calculate the standard deviation

$\theta_{m}: \quad$ The value of threshold for the $m$ th approach

$\theta_{4,1}$ : The value of lower bound for the fourth pothole detection approach

$\theta_{4,2}$ : The value of upper bound for the fourth pothole detection approach.

\section{Conflict of Interests}

The authors declare that there is no conflict of interests regarding the publication of this paper.

\section{Acknowledgment}

The research is supported by the National Science Council of Taiwan under Grants nos. NSC 102-2622-H-009-001CC3, NSC102-2410-H146-002-MY2, NSC 102-2410-H-009052-MY3, and MOST 103-2622-H-009-001-CC3.

\section{References}

[1] Ministry of Justice, National Compensation Statistics, and Statistics of Justice, 2012, http://www.moj.gov.tw/ct.asp?xItem $=36988 \&$ CtNode $=11625 \& \mathrm{mp}=095$.

[2] E. Miluzzo, N. D. Lane, K. Fodor et al., "Sensing meets mobile social networks: The design, implementation and evaluation of the CenceMe application," in Proceedings of the 6th ACM Conference on Embedded Networked Sensor Systems (SenSys '08), pp. 337-350, Raleigh, Calif, USA, November 2008.

[3] C.-C. Lo, C.-H. Chen, D.-Y. Cheng, and H.-Y. Kung, "Ubiquitous healthcare service system with context-awareness capability: design and implementation," Expert Systems with Applications, vol. 38, no. 4, pp. 4416-4436, 2011.

[4] C. I. Wu, H. Y. Kung, C. H. Chen, and L. C. Kuo, "An intelligent slope disaster prediction and monitoring system based on WSN and ANP," Expert Systems with Applications, vol. 41, no. 10, pp. 4554-4562, 2014.
[5] C.-H. Chen, H.-C. Chang, C.-Y. Su, C.-C. Lo, and H.-F. Lin, "Traffic speed estimation based on normal location updates and call arrivals from cellular networks," Simulation Modelling Practice and Theory, vol. 35, no. 1, pp. 26-33, 2013.

[6] X. Yu and E. Salari, "Pavement pothole detection and severity measurement using laser imaging," in Proceedings of the IEEE International Conference on Electro/Information Technology (EIT '11), pp. 1-5, Mankato, Minn, USA, May 2011.

[7] J. Lin and Y. Liu, "Potholes detection based on SVM in the pavement distress image," in Proceedings of the 9th International Symposium on Distributed Computing and Applications to Business, Engineering and Science (DCABES '10), pp. 544-547, Hong Kong, August 2010.

[8] K. de Zoysa, C. Keppitiyagama, G. P. Seneviratne, and W. W. A. T. Shihan, "A public transport system based sensor network for road surface condition monitoring," in Proceedings of the Workshop on Networked Systems for Developing Regions, Kyoto, Japan, August 2007.

[9] J. Eriksson, L. Girod, B. Hull, R. Newton, S. Madden, and H. Balakrishnan, "The pothole patrol: using a mobile sensor network for road surface monitoring," in Proceedings of the 6th International Conference on Mobile Systems, Applications, and Services (MobiSys '08), pp. 29-39, June 2008.

[10] P. Mohan, V. N. Padmanabhan, and R. Ramjee, "Nericell: rich monitoring of road and traffic conditions using mobile smartphones," in Proceedings of the 6th ACM Conference on Embedded Network Sensor Systems, Raleigh, NC, USA, 2008.

[11] A. Mednis, G. Strazdins, R. Zviedris, G. Kanonirs, and L. Selavo, "Real time pothole detection using Android smartphones with accelerometers," in Proceedings of the International Conference on Distributed Computing in Sensor Systems (DCOSS '11), pp. 16, IEEE, Barcelona, Spain, June 2011.

[12] R. Zviedris, A. Elsts, G. Strazdins, A. Mednis, and L. Selavo, "LynxNet: wild animal monitoring using sensor networks," in Proceedings of the 4th International Conference on Real-world Wireless Sensor Networks, Colombo, Sri Lanka, 2010.

[13] J. Han and M. Kamber, Data mining: Concepts and Techniques, Morgan Kaufmann, San Francisco, Calif, USA, 2006. 


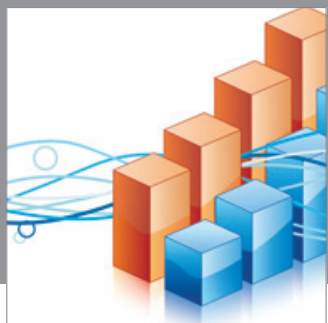

Advances in

Operations Research

mansans

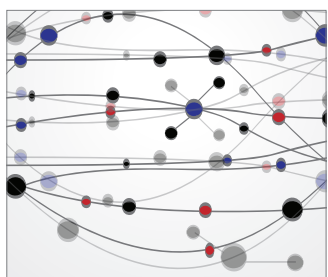

The Scientific World Journal
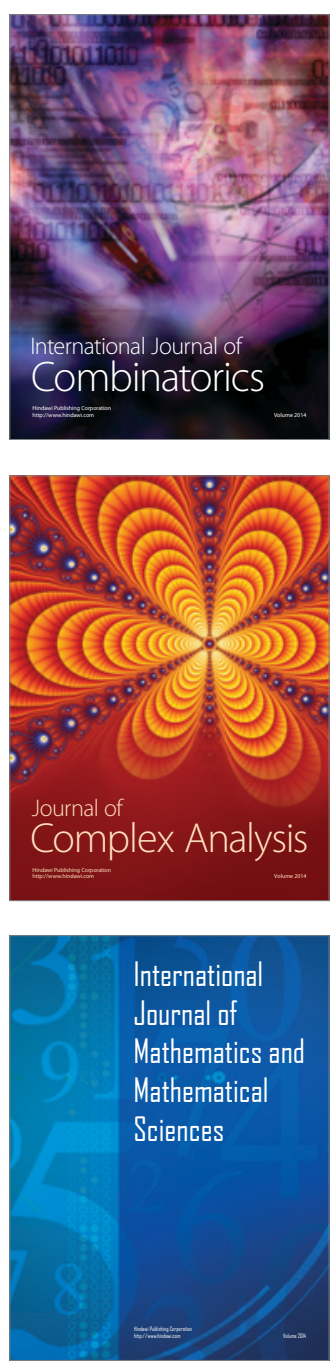
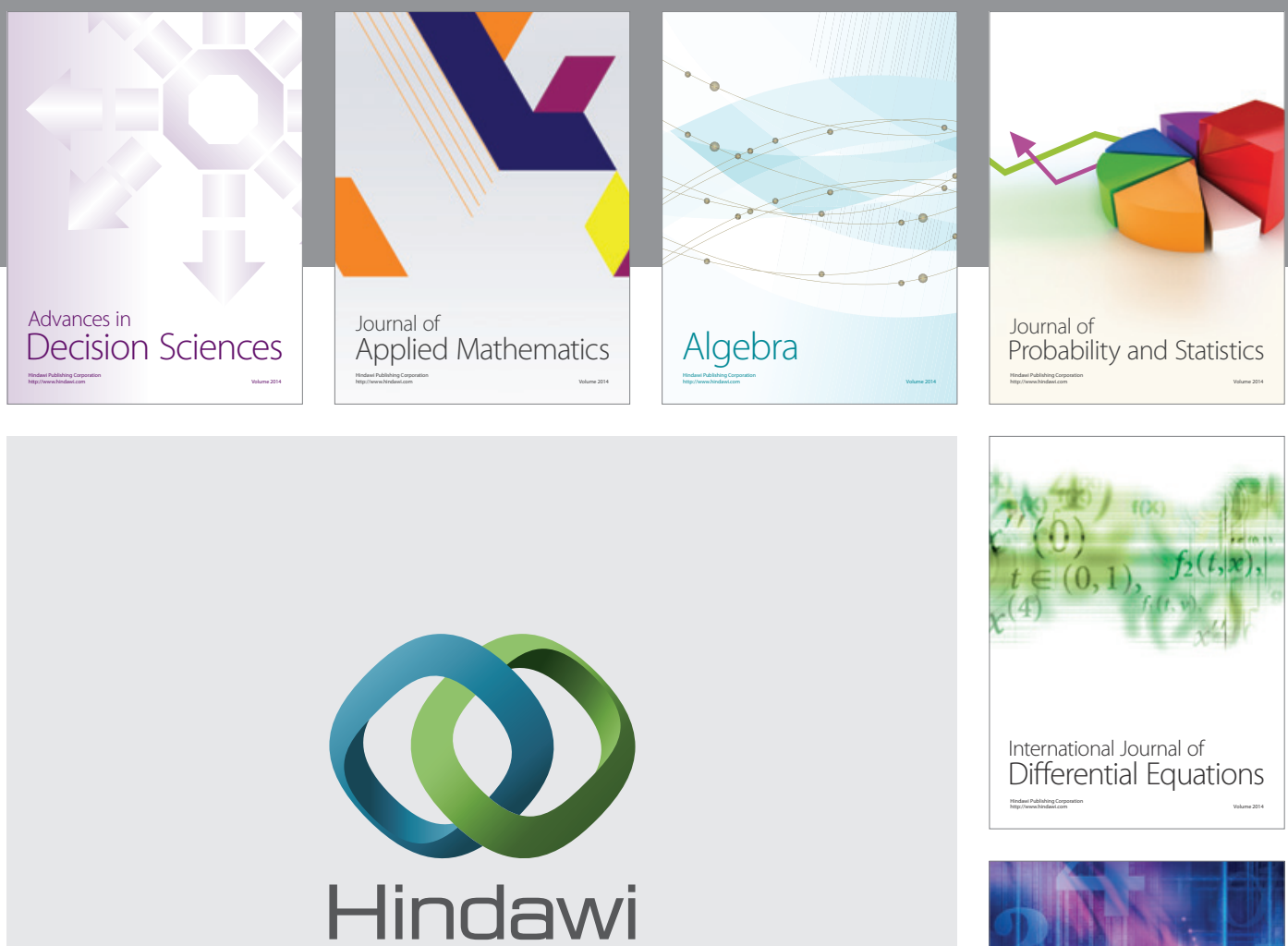

Submit your manuscripts at http://www.hindawi.com
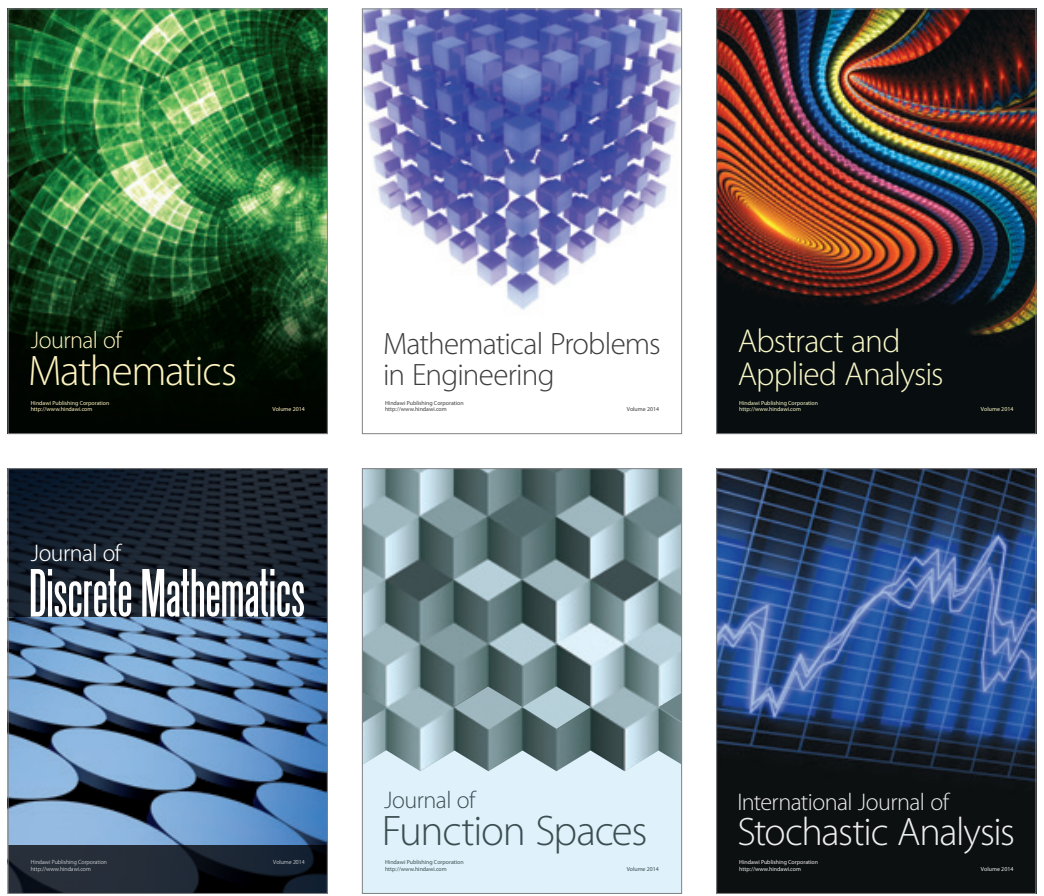

Journal of

Function Spaces

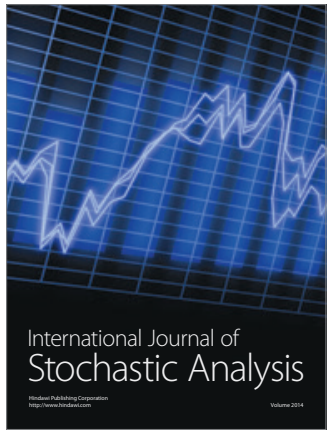

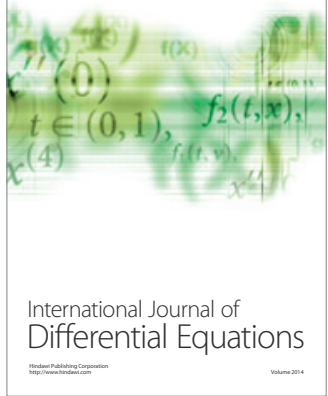
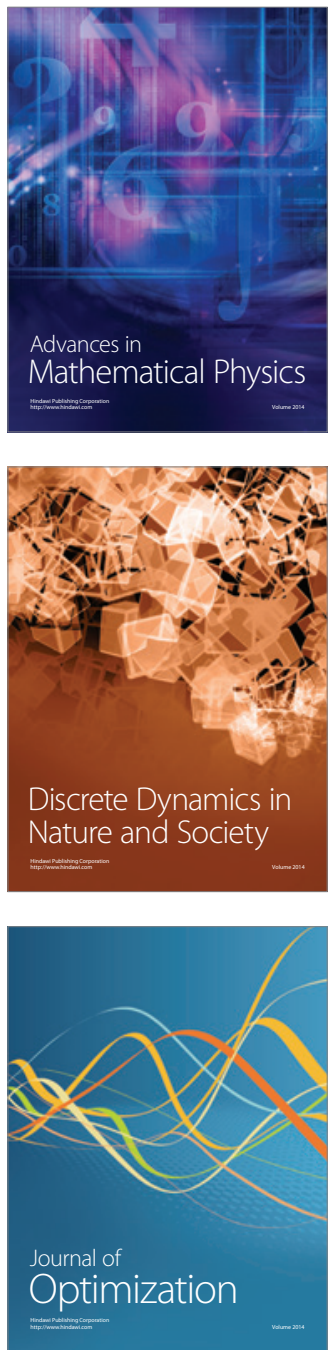